\title{
ISOTYPE SUBMODULES OF $p$-LOCAL BALANCED PROJECTIVE GROUPS
}

\author{
MARK LANE
}

\begin{abstract}
By giving necessary and sufficient conditions for two isotype submodules of a $p$-local balanced projective group to be equivalent, we are able to introduce a general theory of isotype submodules of $p$-local balanced projective groups (or $I B$ modules). Numerous applications of the above result are available particularly for the special class of $I B$ modules introduced by Wick (known as SKT modules). We first show that the class of SKT modules is closed under direct summands, and then we are able to show that if $H$ appears as an isotype submodule of the $p$-local balanced projective group $G$ such that $G / H$ is the coproduct of countably generated torsion groups, then $H$ is an SKT module. Finally we show that $I B$ modules satisfy general structural properties such as transitivity, full transitivity, and the equivalence of $p^{\alpha}$-high submodules.
\end{abstract}

1. Introduction. The recent developments in the study of summands of mixed simply presented groups have generated answers to old problems, introduced new techniques for study of mixed abelian groups, and initiated new problems and conjectures along with interesting parallels to the study of simply presented $p$ groups. Much of the success attributed above has been achieved by formulating the proper types of subgroups so that a third axiom of countability (or Axiom 3) characterization of the aforementioned mixed groups is possible (see $[\mathbf{9}, \mathbf{5}, \mathbf{6}$, and 11]). The restricted study of $p$-local groups (that is, modules over the ring $Z_{p}$ of integers localized at a fixed prime $p$ ) has provided a proving ground for the more complicated global setting, but it has turned out that the global analogues are not formulated so simply in terms of well-known notions as in the local case. Moreover, certain structure theorems are possible for $p$-local groups that do not generalize to the global case, and so study of $p$-local groups is of separate interest. In this note we will investigate isotype submodules of $p$-local balanced projective groups (or $I B$ modules). This topic initially appeared in [10], and the results developed there were used to obtain a characterization of the balanced projective dimension of $p$-local abelian groups, when that dimension is finite.

All groups and modules in this paper will be assumed to be $p$-local and abelian. A subgroup $H$ of $G$ is said to be isotype provided $p^{\alpha} H=p^{\alpha} G \cap H$ for each ordinal $\alpha$ and nice if $p^{\alpha}(G / H)=p^{\alpha} G+H / H$. If $H$ satisfies both of the properties mentioned above, then $H$ is said to be balanced and gives rise to the balanced short exact

Received by the editors March 14, 1986. The contents of this paper represent part of a talk the author gave at an abelian groups workshop sponsored by the University of Colorado at Colorado Springs, May 26-31, 1986.

1980 Mathematics Subject Classification (1985 Revision). Primary 20K21, 20K10, 20K30, $20 \mathrm{~K} 27$.

Key words and phrases. Balanced projective groups, isotype subgroups, $c$-valuation, equivalence, SKT modules, cotorsion hull. 
sequence $0 \rightarrow H \rightarrow G \rightarrow G / H \rightarrow 0$. A group $G$ is said to be balanced projective provided it satisfies the projective property with respect to all balanced short exact sequences. The torsion balanced projectives are simply the totally projective groups, and the class of isotype subgroups of totally projective groups (or IT groups) was investigated in [3 and 4]. It is the appearance of an Axiom 3 characterization of balanced projective groups [9] and of Theorem 1.1 below which suggests that the ideas in [4] can be extended to yield results on the structure of $I B$ modules.

As usual in the study of simply presented groups, the notion of height will be of central importance. Recall that if $G$ is a $p$-local group, then $x \in G$ has height $\alpha$ provided $x \in p^{\alpha} G \backslash p^{\alpha+1} G$, and $x$ has height $\infty$ if $x \in p^{\alpha} G$ for all ordinals $\alpha$. We will write $|x|_{G}$ to denote the height of $x$ computed in $G$. More generally, we can view the height function as a valuation and view $G$ as a valuated group [12]. We will deviate slightly from [12] and use Hill and Megibben's treatment of valuations [4]. If $G$ is a $p$-local group, a valuation $|*|$ on $G$ is a function from $G$ to the set of ordinals adjoined with $\infty$ satisfying the following properties:

(1) $|0|=\infty$ and $|n x|=|x|$ if $(n, p)=1$,

(2) $|x+y| \geq \min \{|x|,|y|\}$, and

(3) $|p x| \geq|x|$ and $|p x|>|x|$ if $|x|$ is an isolated ordinal.

Two immediate consequences of the foregoing definition are $|x+y|=\min \{|x|,|y|\}$ whenever $|x| \neq|y|$ and for any ordinal $\alpha$, there is associated a subgroup $G(\alpha)=$ $\{x \in G:|x| \geq \alpha\}$. A valuated group $G$ is said to be $c$-valuated [4] provided the valuation, in addition to properties (1)-(3), satisfies

(4) $|x| \geq 1$ for all $x$ and if $|x|>\alpha \geq 1$ with $\alpha$ an isolated ordinal, then $x=p y$ for some $y \in A$ with $|y| \geq \alpha$.

This latter condition can be summarized as $G=G(1)$ and $G(\alpha+1) \subseteq p G(\alpha)$ whenever $\alpha \geq 1$ is isolated.

The theory of $c$-valuated groups is explored in some depth in [4], and it will be necessary to highlight the results especially important to our study. Every subgroup $B$ of a $c$-valuated group $G$ is valuated by restriction, and $B$ is itself $c$ valuated (and is said to be a $c$-valuated subgroup) if $B \cap G(\alpha+1) \subseteq p(B \cap G(\alpha)$ ) for all isolated ordinals $\alpha \geq 1$. The quotient $G / B$ is always $c$-valuated by the induced $c$-valuation $|x+B|=\sup _{b \in B}|x+b|$. Any $p$-local group $G$ is $c$-valuated by the maximum valuation $|x|=|x|_{G}+1$, and the $c$-valuation on $G / B$ induced by this $c$-valuation will be given the special name of coset valuation. The following fundamental result from [4] illuminates the close relationship between the general category of $c$-valuated groups and the theory of $I B$ modules.

THEOREM 1.1 (HILl AND MEGIBBEN). If $A$ is any c-valuated group, then there exists an isotype submodule $H$ of a p-local balanced projective group $G$ such that $A \cong G / H$ by a map preserving c-valuations when $G / H$ is endowed with the coset valuation.

A subgroup $K$ of a $p$-local group $G$ is $\aleph_{0}$-separable $[\mathbf{3}]$ provided the coset valuation on $G / K$ never assumes limit values of cofinality greater than $\omega$. If $G$ is a group of length $\tau$ and $H$ is a subgroup of $G$, then an ascending chain $H \subseteq K_{1} \subseteq \cdots \subseteq$ $K_{\alpha} \subseteq \cdots \subseteq G$, where $K_{\alpha}$ is $\aleph_{0}$-separable in $G$ with $K_{\alpha+1} / K_{\alpha}$ countable for each $\alpha, K_{\beta}=\bigcup_{\alpha<\beta} K_{\alpha}$ whenever $\beta$ is a limit ordinal, and $G=\bigcup_{\alpha<\tau} K_{\alpha}$, is called a 
composition series of $\aleph_{0}$-separable submodules over $H$. The following theorem is the highlight of the early development of the theory of $I B$ modules.

THEOREM $1.2[\mathbf{1 0}]$. If $H$ is an isotype submodule of the p-local balanced projective group $G$, then $H$ is itself balanced projective if and only if $G$ has a composition series of $\aleph_{0}$-separable submodules over $H$.

We say that a direct sum $\bigoplus_{I} A_{i}$ is a valuated coproduct (or simply a coproduct) if $\left|\sum_{I} a_{i}\right|=\min _{I}\left|a_{i}\right|$ whenever each $a_{i} \in A_{i}$ and only finitely many of the terms in the sum are nonzero. If $\left\{x_{i}\right\}_{I}$ is an independent set of elements of infinite order generating a valuated coproduct such that $\left|p^{k} x_{i}\right| \leq\left|x_{i}\right|+k$ for each $i$ and each $k<\omega$, then we say that $\bigoplus_{I}\left\langle x_{i}\right\rangle$ is a free valuated subgroup, and if $\left|x_{i}\right|=\left|x_{i}\right|_{G}$, then the inequality $\leq$ can be replaced by $=$. If a $p$-local group $G$ has such a set $X=\left\{x_{i}\right\}_{I}$ with $G /\langle X\rangle$ torsion, then $X$ is called a $K$-basis for $G$. More generally, $G$ is said to be a $K$-module provided every finitely generated submodule is a finite extension of a (necessarily finitely generated) free valuated submodule. Following [9], we say that a submodule $N$ of $G$ is $K$-nice if $N$ is nice and $G / N$ is a $K$-module. It was shown in the preceding reference that $G$ is balanced projective if and only if $G$ satisfies Axiom 3 with respect to $K$-nice submodules. Moreover, this condition is equivalent to $G$ possessing a $K$-basis and satisfying Axiom 3 with respect to nice submodules. It was shown in $[\mathbf{1 0}]$ that $G$ is balanced projective if and only if there exists a $K$-nice composition series for $G$; that is an ascending chain

$$
0=N_{0} \subseteq N_{1} \subseteq \cdots \subseteq N_{\alpha} \subseteq \cdots \subseteq G
$$

of $K$-nice submodules with $N_{\alpha+1} / N_{\alpha}$ countable for each ordinal $\alpha, N_{\beta}=\bigcup_{\alpha<\beta} N_{\alpha}$ for all limit ordinals $\beta$, and $G=\bigcup_{\alpha<\tau} N_{\alpha}$ for $\tau$ the length of $G$.

Following Warfield [16], we say that a $p$-local group $M$ is a $\lambda$-elementary balanced projective group (where $\lambda$ is a limit ordinal) if $p^{\lambda} M \cong Z_{p}$ and $M / p^{\lambda} M$ is torsion totally projective. Under a careful correlation between uniqueness and existence theorems for balanced projectives, Warfield was able to show that every balanced projective can be written as a direct sum of $\lambda$-elementary balanced projective groups for various limit ordinals $\lambda$, a totally projective group, and a divisible group. One of the consequences of this is that $G$ has a $K$-basis $X$, and following [7] we realize that $\langle X\rangle$ is nice in $G$ with $G /\langle X\rangle$ totally projective.

The theory of balanced projective groups has become one of the most satisfactory in the study of mixed groups since they are classified by a complete set of invariants and admit the above three distinct yet equivalent descriptions. Each of these descriptions will be used in varying degrees in the results which follow. In $\S 2$ we present the "main theorem" for $I B$ modules and related results. For $H$ and $K$ isotype submodules of a balanced projective group $G$, we are able to formulate necessary and sufficient conditions for $H$ to be mapped onto $K$ by an automorphism of $G$. We present some applications of this result in $\S 3$ by focusing on a special class of $I B$ modules, and we close by listing some properties that are common to all $I B$ modules in $\S 4$.

2. The equivalence theorem for $I B$ modules. Two submodules $H$ and $K$ of a group $G$ are said to be equivalent if there is an automorphism of $G$ mapping $H$ onto $K$. The notion of equivalence has received a lot of attention in the literature, and Theorem 3.1 in [4], which states necessary and sufficient conditions for 
two isotype subgroups of a totally projective $p$-group to be equivalent, has had a profound impact on the study of $I T$ groups. We have generalized that theorem to $I B$ modules, and several applications are available in the same spirit. Our proof includes a revision of the torsion analogue since it is important for the reader to see that our generalization is natural.

For $G$ a $p$-local group, the $\alpha$ th Ulm-Kaplansky invariant of $G$ is written $f_{G}(\alpha)$ and defined to be $\operatorname{dim}\left[p^{\alpha} G[p] / p^{\alpha+1} G[p]\right]$, and if $\lambda$ is a limit ordinal, the $\lambda \operatorname{th}$ Warfield $h$-invariant of $G$ is written $h_{G}(\lambda)$ and defined as $\operatorname{dim}\left[p^{\lambda} G /\left(p^{\lambda+1} G+T_{\lambda}\right)\right]$, where $T_{\lambda}$ represents the torsion part of $p^{\lambda} G$. Divisible $p$-local groups can be classified by the invariants $f_{G}(\infty)=\operatorname{dim} p^{\infty} G[p]$ and $h_{G}(\infty)=\operatorname{dim}\left[p^{\infty} G \otimes Q\right]$, where $Q$ is the quotient field of $Z_{p}$. Totally projective groups are determined uniquely by their Ulm-Kaplansky invariants [2], and balanced projective groups are classified by the Ulm-Kaplansky and Warfield $h$-invariants [16]. The author [9] has proved the latter statement by using the same techniques Hill used in [2], and so both results can be resolved by considering relative invariants. The notation used in [14] and [9] is especially fruitful in our development. Suppose $A$ is a submodule of the $p$-local group $G, \alpha$ is an ordinal, and $\lambda$ is a limit ordinal, and denote

$$
A(\alpha)=\left(p^{\alpha+1} G+A\right) \cap p^{\alpha} G[p]
$$

and

$$
A_{\lambda+n}=p^{\lambda+n} G /\left[\left(p^{\lambda+n+1} G+A+T_{\lambda+n}\right) \cap p^{\lambda+n} G\right]
$$

for $n$ a nonnegative integer. Then the $\alpha$ th Ulm-Kaplansky invariant of $G$ relative to $A$ and the $\lambda$ th Warfield $h$-invariant of $G$ relative to $A$ are defined as $f_{G}(\alpha, A)=$ $\operatorname{dim}\left[p^{\alpha} G[p] / A(\alpha)\right]$ and $h_{G}(\lambda, A)=\operatorname{dim}\left[\lim p^{\lambda+n} G / A_{\lambda+n}\right]$, respectively.

THEOREM 2.1. Isotype submodules $H$ and $K$ of the balanced projective module $G$ are equivalent if and only if the following three conditions are satisfied:

(1) $H$ and $K$ have the same Ulm-Kaplansky invariants,

(2) $H$ and $K$ have the same Warfield $h$-invariants,

(3) $G / H \cong G / K$ by a map preserving coset valuations.

The relevance of the theory of $c$-valuated groups to the study of $I B$ modules is now evident through Theorem 1.1 and Theorem 2.1. It is the abstracted properties of the coset valuation that display all of the information about the structure of an isotype submodule of a balanced projective beyond the Ulm-Kaplansky and Warfield $h$-invariants. We will actually prove the following generalization of Theorem 2.1.

THEOREM 2.2. Let $A$ and $B$ be $K$-nice submodules of the p-local groups $G$ and $G^{\prime}$, respectively, such that both $G / A$ and $G^{\prime} / B$ are balanced projective, and suppose $H$ and $K$ are isotype submodules of $G$ and $G^{\prime}$ containing $A$ and $B$, respectively. Then each height preserving isomorphism $\tau: A \rightarrow B$ extends to an isomorphism $\psi$ from $G$ to $G^{\prime}$ with $\psi(H)=K$ if and only if the Ulm-Kaplansky invariants of $H$ relative to $A$ and of $K$ relative to $B$ agree, the Warfield $h$-invariants of $H$ relative to $A$ and of $K$ relative to $B$ agree, and there is an isomorphism $\phi: G / H \rightarrow G^{\prime} / K$ preserving coset valuations. Moreover, if the stated conditions are satisfied, then $\psi$ can be chosen so that it induces $\phi$.

PROOF. Since the three stated conditions are clearly necessary, we will assume they are satisfied and will establish the existence of the desired extension $\psi$ of $\tau$. 
We begin by defining for arbitrary submodules $M \subseteq G$ and $N \subseteq G^{\prime}$ the following submodules for each ordinal $\alpha$ :

$$
\begin{aligned}
& M_{H}(\alpha)=\left(p^{\alpha+1} G+M\right) \cap p^{\alpha} H[p], \\
& N_{K}(\alpha)=\left(p^{\alpha+1} G^{\prime}+N\right) \cap p^{\alpha} K[p], \\
& M_{\alpha}^{H}=\left(p^{\alpha+1} G+M+T_{p^{\alpha} H}\right) \cap p^{\alpha} H, \\
& N_{\alpha}^{K}=\left(p^{\alpha+1} G^{\prime}+N+T_{p^{\alpha} K}\right) \cap p^{\alpha} K .
\end{aligned}
$$

The notation $T_{p^{\alpha} H}$ and $T_{p^{\alpha} K}$ will be used to denote the torsion parts of $p^{\alpha} H$ and $p^{\alpha} K$, respectively. The key to proving this theorem is in the family $\theta$ of all triples $(M, N, \pi)$, where $M$ and $N$ are $K$-nice submodules of $G$ and $G^{\prime}$ containing $A$ and $B$, respectively, and satisfying the following four conditions:

(i) $\pi: M \rightarrow N$ is a height preserving isomorphism extending $\tau$,

(ii) $\pi(x)+K=\phi(x+H)$ for all $x \in M$,

(iii) $p^{\alpha} H[p] / M_{H}(\alpha) \cong p^{\alpha} K[p] / N_{K}(\alpha)$ for all ordinals $\alpha$, and

(iv) $\underset{\lim }{\longrightarrow} p^{\lambda+n} H / M_{\lambda+n}^{H} \cong \lim _{\longrightarrow} p^{\lambda+n} K / N_{\lambda+n}^{K}$ for all limit ordinals $\lambda$.

Observe that $(A, B, \tau) \in \theta$ since $H$ and $K$ are isotype in $G$ and $G^{\prime}$ and contain $A$ and $B$, respectively, and since the relative invariants of $H$ to $A$ and of $K$ to $B$ agree. Recalling that $G / A$ and $G^{\prime} / B$ are balanced projective and remembering the argument used in the proof of the uniqueness theorem for balanced projectives exploiting Axiom $3[9]$, it is clear that we need only prove that for any extension $M^{\prime}=\langle M, y\rangle$ of $M$ with $(M, N, \pi) \in \theta$, there is a triple $\left(M^{\prime}, N^{\prime}, \pi^{\prime}\right)$ in $\theta$ for some appropriate $N^{\prime} \subseteq G^{\prime}$ with $\pi^{\prime}$ extending $\pi$. The proof of this takes on two mutually distinct cases: when $y+M$ has finite order and when $y+M$ has infinite order.

Case 1. Suppose $y+M$ has finite order with $|y|_{G}=\alpha$. Since $M$ is nice in $G$, we can assume that $p y \in M$ and $y$ is proper with respect to $M$. We will obtain the desired extension $\pi^{\prime}$ of $\pi$ by finding a $y^{\prime} \in G^{\prime}$ satisfying the following five conditions:

(1) $\left|y^{\prime}\right|_{G^{\prime}}=\alpha$,

(2) $p y^{\prime}=\pi(p y)$,

(3) $y^{\prime}$ is proper with respect to $N$,

(4) $y^{\prime}+K=\phi(y+H)$, and

(5) there is an isomorphism $M_{H}^{\prime}(\beta) / M_{H}(\beta) \rightarrow N_{K}^{\prime}(\beta) / N_{K}(\beta)$ for all ordinals $\beta$, which is induced from (iii).

Two separate cases are considered.

Case a. Suppose $|p y|_{G}>\alpha+1$ and $|y+H|>\alpha+1$, where $|y+H|$ denotes the coset valuation on $G / H$. Since $\phi$ preserves coset valuations, $\phi(y+H)=w+K$ where $|w|_{G^{\prime}}>\alpha$. By (ii), it follows that $p w-\pi(p y) \in K \cap p^{\alpha+2} G^{\prime}=p^{\alpha+2} K$. By replacing $w$ by $w+k$ for some $k \in K$ if necessary, we are able to assume that $p w=\pi(p y)$. Since $|y+H|>\alpha+1$, there must exist some $h \in H$ such that $|y+h|_{G}>\alpha$, and so $|h|_{G}=\alpha$ and $|p h|_{G}>\alpha+1$. Thus $p h=p h^{\prime}$ for some $h^{\prime} \in p^{\alpha+1} H$. We claim that $h-h^{\prime} \in p^{\alpha} H[p] \backslash\left(p^{\alpha+1} G+M\right)$; for if this is not the case, then we could write $h-h^{\prime}=g^{\prime}+m$ for $g^{\prime} \in p^{\alpha+1} G$ and $m \in M$. But then $h=\bar{g}^{\prime}+m$, where $\bar{g}^{\prime}=g^{\prime}+h^{\prime} \in p^{\alpha+1} G$, and so $|y+m|_{G}=|y+h+m-h|_{G}>\alpha$, and this contradicts $y$ being proper with respect to $M$ with $|y|_{G}=\alpha$. Now there exists a $v \in p^{\alpha+1} G$ such that $s=y-v \in p^{\alpha} H[p] \backslash\left(p^{\alpha+1} G+M\right)$ because of the above claim and the fact that $|y+h|_{G}>\alpha$, and this implies that $y+h-h^{\prime}=v \in p^{\alpha+1} G$. Hence, by condition (iii), there exists some $s^{\prime} \in p^{\alpha} K[p] \backslash\left(p^{\alpha+1} G^{\prime}+N\right)$. Now choose 
$y^{\prime}=w+s^{\prime}$ and observe that (1), (2), and (4) hold for this choice of $y^{\prime}$. Since $y$ is proper with respect to $M$ and $v \in p^{\alpha+1} G, s$ is also proper with respect to $M$. By (iii) again we have that $s^{\prime}$ is proper with respect to $N=\pi(M)$, and so $y^{\prime}$ satisfies (3) since $w \in p^{\alpha+1} G^{\prime}$. Finally (5) holds since if $\beta=\alpha$, the quotient in (5) is cyclic of order $p$, and if $\beta \neq \alpha$, the quotients in (5) are trivial.

Case b. Suppose that $|p y|_{G}=\alpha+1$ or $|y+H|=\alpha+1$. Since $\phi$ preserves coset valuations, $\phi(y+H)=w+K$ for some $w \in p^{\alpha} G^{\prime}$. By (ii), $p w-\pi(p y) \in K \cap p^{\alpha+1} G^{\prime}$ $=p^{\alpha+1} K$, which implies that $p(w+k)=\pi(p y)$ for some $k \in p^{\alpha} K$. This time we simply take $y^{\prime}=w+k$ so that at least (2) and (4) are satisfied. Since $y^{\prime} \in p^{\alpha} G^{\prime}$, it follows that $|p y|_{G}=|\pi(p y)|_{G^{\prime}}=\left|p y^{\prime}\right|_{G^{\prime}}$ and $|y+H|=|\phi(y+H)|=\left|y^{\prime}+K\right| \geq\left|y^{\prime}\right|_{G^{\prime}}$. Thus for either of the conditions defining Case b, it will follow that $\left|y^{\prime}\right|_{G^{\prime}}=\alpha$ (which is (1)). Now if $y^{\prime}$ fails to be proper with respect to $N$, then we can return to Case a; for if $\left|y^{\prime}+\pi(x)\right|_{G^{\prime}}>\alpha$ with $x \in M$, then $y_{1}=y+x$ is an element of $M^{\prime}$ such that $\left|y_{1}\right|_{G}=\alpha,\left|p y_{1}\right|_{G}>\alpha+1$, and $\left|y_{1}+H\right|>\alpha+1$. Thus we may assume that $y^{\prime}$ satisfies (3), and so it remains to prove (5). But $y^{\prime}$ satisfies (5) vacuously since if this is not the case, then we are also able to return to Case a.

Case 2. Let $y+M$ have infinite order, and assume without loss of generality that $|y|_{G}=\lambda$, a limit ordinal. Since $(\langle y\rangle \oplus M) / M$ is a finitely generated submodule of $G / M$ with $M K$-nice in $G$, there must be a valuated coproduct $\langle\bar{y}\rangle \oplus M$ containing a multiple of $y$ with $\left|p^{n} \bar{y}\right|_{G}=|\bar{y}|_{G}+n$ for each $n<\omega$. By Case 1 , we may replace $y$ by $\bar{y}$ if necessary, and as in that case, we obtain the desired extension $\pi^{\prime}$ of $\pi$ by finding a $y^{\prime} \in G$ satisfying the following four conditions:

(1) $\left|y^{\prime}\right|_{G^{\prime}}=\lambda$,

(2) $\left\langle y^{\prime}\right\rangle \oplus N$ is a valuated coproduct with $\left|p^{n} y^{\prime}\right|_{G^{\prime}}=\lambda+n$ for each $n<\omega$,

(3) $y^{\prime}+K=\phi(y+H)$, and

(4) there is an induced isomorphism $\left[M^{\prime} / M\right]_{\gamma}^{H} \cong\left[N^{\prime} / N\right]_{\gamma}^{K}$ for all limit ordinals $\gamma$, where $\left[M^{\prime} / M\right]_{\gamma}^{H}$ denotes the direct limit $\underline{\lim }\left(M^{\prime}\right)_{\gamma+n}^{H} / M_{\gamma+n}^{H}$. The proof of this case will also be separated into two subcases.

Case c. Suppose $|y+H|>\lambda+1$. There must be some $h \in H$ such that $|y-h|_{G} \geq \lambda+1$. Hence $y-h=g \in p^{\lambda+1} G$, and so $h \in p^{\lambda} G \cap H=p^{\lambda} H$. Now we claim that $p^{n} h \notin M_{\lambda+n}^{H}$ for any $n<\omega$. If $p^{n} h=\bar{g}+m$ for some $\bar{g} \in p^{\lambda+n+1} G$ and $m \in M$, then $p^{n} y-m \in p^{\lambda+n+1} G$ which contradicts $\langle y\rangle \oplus M$ being a valuated coproduct with $\left|p^{n} y\right|_{G}=\lambda+n$ for each $n<\omega$. Now the image of $h$ under the natural map $p^{\lambda} H \rightarrow \lim p^{\lambda+n} H / M_{\lambda+n}^{H}$ is nonzero, and so by (iv), there must be a nonzero element of $\underset{\lim }{\longrightarrow} p^{\lambda+n} K / N_{\lambda+n}^{K}$ which is the image of some $k \in p^{\lambda} K$ under the natural map $p^{\lambda K} \rightarrow \stackrel{\lim }{\longrightarrow} p^{\lambda+n} K / N_{\lambda+n}^{K}$. Hence $p^{n} k \notin N_{\lambda+n}^{K}$ for any nonnegative integer $n$. Now suppose that $\phi(g+H)=g_{1}+K$, and note that $\lambda+1 \leq|g|_{G}<|g+H|=\left|g_{1}+K\right|$. Since $\lambda+1$ is an isolated ordinal, there exists some $k_{1} \in K$ such that $g_{1}+k_{1} \in p^{\lambda+1} G^{\prime}$. Set $y^{\prime}=g_{1}+k_{1}+k$. It is easy to see that (3) holds for this choice of $y^{\prime}$ since $\phi(y+H)=\phi(g+H)=g_{1}+K=y^{\prime}+K$. Moreover, since $g_{1}+k_{1} \in p^{\lambda+1} G^{\prime}$ and $|k|_{G^{\prime}}=\lambda$, it follows that $\left|y^{\prime}\right|_{G^{\prime}}=\left|\left(g_{1}+k_{1}\right)+k\right|_{G^{\prime}}=\lambda$, which is (1). If $\left|p^{n} y^{\prime}+z\right| \geq \lambda+n+1$ for some $n<\omega$ and some $z \in N$, then $p^{n} y^{\prime}+z=g^{\prime} \in p^{\lambda+n+1} G^{\prime}$, and this implies that $p^{n} k=\left[g^{\prime}+p^{n}\left(g_{1}+k_{1}\right)\right]-z \in N_{\lambda+n}^{K}$, which contradicts the choice of $k$. Hence $y^{\prime}$ satisfies (2), and we need to argue that (4) holds. When $\beta=\lambda$, then $\left[M^{\prime} / M\right]_{\lambda}^{H}$ and $\left[N^{\prime} / N\right]_{\lambda}^{K}$ are both cyclic of order $p$, 
and so the isomorphism is induced as desired, and when $\beta \neq \lambda$, then both $\left[M^{\prime} / M\right]_{\beta}^{H}$ and $\left[N^{\prime} / N\right]_{\beta}^{K}$ are trivial.

Case d. Suppose that $|y+H|=\lambda+1$ (which says that $y$ is proper with respect to $H$ ). By (ii), we can choose $w$ such that $|w|_{G^{\prime}}=\lambda$ and $\phi(y+H)=w+K$. Now suppose $y^{\prime}=w$ does not have all the desired properties (1)-(4). The only possible problem is for $(\langle w\rangle \oplus N) / N$ not to be a free valuated submodule of $G^{\prime} / N$. Thus $\left|p^{n}+z\right| \geq \lambda+n m+1$ for some $n<\omega$ and some $z \in N$. Now there exists a $g \in p^{\lambda+1} G$ and (since $\left.|y+H|=\lambda+1\right)$ an $h \in p^{\lambda} H$ such that $p^{n}(y+g+h) \in M$, and it is not difficult to see that $p^{r} h \notin M_{\lambda+r}^{H}$ for each $r<\omega$ (since otherwise we would contradict our choice of $y$ because $g \in p^{\lambda+1} G$ ). Hence there exists some $k \in p^{\lambda} K$ such that $p^{r} k \notin N_{\lambda+r}^{K}$ for each $r<\omega$, and we replace $w$ by $w+k$. Now $\langle w+k\rangle \oplus N$ is a valuated coproduct with $\left|p^{m}(w+k)\right|_{G^{\prime}}=\lambda+m$ for each $m<\omega$ since $p^{n} w+z \in p^{\lambda+n+1} G^{\prime}$ and $p^{r} k \notin N_{\lambda+r}^{K}$ for each $r<\omega$. Since $\phi(y+H)=w+k+K$, the proof is complete.

It is often the case that the conditions of Theorem 2.2 stipulating that $H$ and $K$ have the same Ulm-Kaplansky and Warfield $h$-invariants either cannot be assumed or cannot easily be checked. Nevertheless, if $G / H$ and $G^{\prime} / K$ are isomorphic under a map preserving coset valuations, then some information can still be obtained which relates $H$ to $K$. We will call $H$ and $K$ similar if there exists a balanced projective group $B$ such that $H \oplus B \cong K \oplus B$.

COROLlaRY 2.3. If $H$ and $K$ are isotype submodules of the p-local balanced projective groups $G$ and $G^{\prime}$, respectively, and if $G / H \cong G^{\prime} / K$ as valuated groups endowed with the coset valuation, then $H$ and $K$ are similar.

ProOF. We may assume that both $G$ and $G^{\prime}$ are reduced. Let $\mu$ be a limit ordinal greater than the lengths of $G$ and $G^{\prime}$, and let $\aleph_{\mu}=\max \left\{\aleph_{0}\right.$, cardinal associated with $\mu$ \}. Now define two functions $f$ and $h$ from the ordinals to the cardinals as follows:

and

$$
f(\alpha)= \begin{cases}\aleph_{\mu} & \text { if } \alpha<\mu, \\ 0 & \text { otherwise }\end{cases}
$$

$$
h(\alpha)= \begin{cases}\aleph_{\mu} & \text { if } \alpha \text { is a limit ordinal }<\mu, \\ 0 & \text { otherwise. }\end{cases}
$$

By Warfield's definition [15], $(f, h)$ is an admissible pair, and so by Theorem 4.1 in that paper there exists a balanced projective group $B$ with Ulm-Kaplansky invariants $f$ and Warfield $h$-invariants $h$. Hence $H \oplus B \cong K \oplus B$ by Theorem 2.2.

When isomorphism is not required in Theorem 2.2 , the hypotheses can be weakened and the argument needed is not nearly as delicate.

THEOREM 2.4. Suppose $A$ and $H$ are submodules of the p-local group $G$ such that $A \subseteq H, A$ is $K$-nice in $G$, and $G / A$ is balanced projective. If $K$ is an isotype submodule of the p-local group $G^{\prime}$ and if there exists a homomorphism $\phi: G / H \rightarrow$ $G^{\prime} / K$ that does not decrease coset valuations, then any homomorphism $\tau: A \rightarrow K$ that does not decrease heights (as computed in $G$ and $G^{\prime}$, respectively) extends to a homomorphism $\psi: G \rightarrow G^{\prime}$ that induces $\phi$.

PROOF. Let $₹$ be the family of all homomorphisms $\psi: N \rightarrow G^{\prime}$ extending $\tau$ that do not decrease heights such that if $C$ is the collection of $K$-nice submodules 
of $G / A$ satisfying Axiom 3, then $N / A \in C$ and $\phi(z+H)=\psi(z)+K$ for all $z \in N$. Zorn's lemma can be applied in the obvious way, and so we can choose a maximal member of $\mathcal{F}$, which we will denote $\psi_{0}: N_{0} \rightarrow G^{\prime}$. If $N_{0} \neq G$, then we can choose $x \in G \backslash N_{0}$ and (by the countable extension property) an $N \subseteq G$ with $N / A \in C$ and $N /\left\langle N_{0}, x\right\rangle$ countable. If $z \in N \backslash N_{0}$ and $z+N_{0}$ has finite order, then without loss of generality we may assume that $p z \in N_{0}$ and $z$ is proper with respect to $N_{0}$. If $\phi(z+H)=y+K$, then $p y-\psi_{0}(p z) \in K \cap p^{\alpha+1} G^{\prime}=p^{\alpha+1} K$, and so by replacing $y$ by $y+\bar{k}$ for some $\bar{k} \in p^{\alpha} K$ if necessary, we may assume that $p y=\psi_{0}(p z)$. Thus $\psi_{1}:\left\langle N_{0}, z\right\rangle \rightarrow K$ defined by $\psi_{1}(w+t z)=\psi_{0}(w)+t y$ for all $t \in Z_{p}$ with $(t, p)=1$ and $w \in N_{0}$ is an extension of $\tau$ which does not decrease heights satisfying $\phi\left(w^{\prime}+H\right)=\psi_{1}\left(w^{\prime}\right)+K$ for all $w^{\prime} \in\left\langle N_{0}, z\right\rangle$. If $z+N_{0}$ has infinite order, then we may assume that $N_{0} \oplus\langle z\rangle$ is a valuated coproduct with $\left|p^{n} z\right|_{G}=|z|_{G}+n$ for each $n<\omega$. If $\phi(z+H)=y+K$, then by choosing $k \in K$ with $|y+k|_{G^{\prime}} \geq|z|_{G}$, it follows that $\psi_{1}:\left\langle N_{0}, z\right\rangle \rightarrow K$ defined by $\psi_{1}(w+t z)=\psi_{0}(w)+t(y+k)$ for all $w \in N_{0}$ and $t \in Z_{p}$ is an extension of $\tau$ which does not decrease heights with $\phi\left(w^{\prime}+H\right)=\psi_{1}\left(w^{\prime}\right)+K$ for each $w^{\prime} \in\left\langle N_{0}, z\right\rangle$. A countable process will ensure that there exists an extension $\psi: N \rightarrow K$ of $\tau$ that does not decrease heights with $\phi(z+H)=\psi(z)+K$ for all $z \in N$. But $N / A \in C$, and so we conclude that $N_{0}=G$.

COROLLARY 2.5. Let $H$ and $K$ be submodules of the balanced projective module $G$ with $K$ isotype in $G$. Then each homomorphism $\phi: G / H \rightarrow G / K$ which does not decrease coset valuations is induced by an endomorphism of $G$.

3. SKT modules. In this section we will present some applications of Theorem 2.2 by considering a special class of $I B$ modules. Following Warfield [15], a $p$-group is said to be an $S$-group if it appears as the torsion part of a $p$-local balanced projective group, and following Wick [17], we say that a $p$-local group $M$ is an $S K T$ module if it is isomorphic to a direct sum of an $S$-group and a balanced projective module. Wick introduced the class of SKT modules as a class of $p$ local groups which contains the balanced projectives and the $S$-groups and can be classified by known invariants. Moreover, SKT modules satisfy a natural projective property which allows one to study them homologically. A short exact sequence $0 \rightarrow A \rightarrow B \rightarrow C \rightarrow 0$ is said to be ch-pure provided both the sequence and the sequence of cotorsion hulls $0 \rightarrow c(A) \rightarrow c(B) \rightarrow c(C) \rightarrow 0$ are balanced short exact sequences. For a reduced module $M$, the cortorsion hull of $M$ is defined as $\operatorname{Ext}\left(Q / Z_{p}, M\right)$ and denoted $c(M)$. The module $c(M)$ is also reduced, and $M$ can be embedded as an isotype submodule with $c(M) / M$ torsion-free and divisible. A $p$ local group $G$ is said to be ch-pure projective if it enjoys the projective property with respect to all ch-pure sequences. Wick [17] has shown that $G$ is ch-pure projective if and only if $G$ is a summand of an SKT module (which may not necessarily be reduced). Later Stanton $[\mathbf{1 3}]$ attempted to prove that all summands of an SKT module are also SKT modules, but his proof (although it contains the right idea) is not correct. Later Hunter and Walker [8] put together an admirably smooth proof that summands of $S$-groups are $S$-groups, but they did not salvage the whole problem. The summand problem of Stanton is important to our development, and so we will present a proof in the spirit of [8]. If $B$ is a submodule of the $p$-local group $A$, then we will denote $[B: A]$ to be the subgroup of all elements $a \in A$ with $p^{k} a \in B$ for some nonnegative integer $k$. 
THEOREM 3.1. Suppose $A$ is a reduced p-local group that has a $K$-basis $X$ with $A /\langle X\rangle$ a (reduced) $S$-group. Then $A$ is an SKT module.

Proof. By Lemma 3 and $\S 2(\mathrm{~B})$ in $[8]$, there is a free valuated submodule $Y$ of $c(A /\langle X\rangle)$ such that $[Y: c(A /\langle X\rangle)] / Y$ is totally projective. Now the reduced part $\bar{A} /\langle X\rangle$ of $c(A) /\langle X\rangle$ contains $A /\langle X\rangle$ and is cotorsion since it is an epimorphic image of the cotorsion group $c(A)$. Hence $c(A /\langle X\rangle) \cong \bar{A} /\langle X\rangle$ since $\bar{A} /\langle X\rangle / A /\langle X\rangle$ is torsion-free and divisible. Thus there exists a free valuated submodule $\bar{Y} /\langle X\rangle$ of $\bar{A} /\langle X\rangle$ with $[\bar{Y} /\langle X\rangle: \bar{A} /\langle X\rangle] / \bar{Y} /\langle X\rangle$ totally projective. But then $[\bar{Y}: \bar{A}] / \bar{Y}$ is totally projective with the extension $\bar{Y}$ of $\langle X\rangle$ splitting since $\langle X\rangle$ is necessarily nice in $\bar{Y}$ by Theorem 8 in [12]. Hence $\bar{Y}=\left\langle Y^{\prime}\right\rangle \oplus\langle X\rangle$, and by Theorem 31 in [7], $\bar{Y}$ is nice in $[\bar{Y}: \bar{A}]$. It follows that $[\bar{Y}: \bar{A}]$ is balanced projective, and so by Warfield's description [16], we can decompose $[\bar{Y}: \bar{A}]=T \oplus \bigoplus_{x \in X} K_{x} \oplus \bigoplus_{x \in Y^{\prime}} K_{x^{\prime}}$ where $K_{x}$ is a $\lambda$-elementary balanced projective module with $\langle x\rangle=p^{\lambda} K_{x}$ for some limit ordinal $\lambda$ and $T$ is a totally projective group. Finally $A=T \oplus \bigoplus_{x \in X} K_{x} \oplus \bigoplus_{x \in Y^{\prime}} T_{x}$, where $T_{x}$ is the torsion part of $K_{x}$, and so $A$ is an SKT module.

\section{COROllary 3.2. A summand of an SKT module is an SKT module.}

Proof. Suppose $M=K \oplus S=A \oplus B$, where $K$ is a balanced projective and $S$ is an $S$-group. We need to show that $A$ and $B$ are SKT modules. By Theorem 6.6 in [1], we may assume there exist $K$-bases $Y_{A}$ for $A$ and $Y_{B}$ for $B$ which are contained in $K$ with $Y_{A} \cup Y_{B}$ a $K$-basis for $K$. But then $A /\left\langle Y_{A}\right\rangle \oplus B /\left\langle Y_{B}\right\rangle \cong$ $K /\left(\left\langle Y_{A}\right\rangle \oplus\left\langle Y_{B}\right\rangle\right) \oplus S$ is an $S$-group since $K /\left(\left\langle Y_{A}\right\rangle \oplus\left\langle Y_{B}\right\rangle\right)$ is totally projective. Hence $A /\left\langle Y_{A}\right\rangle$ and $B /\left\langle Y_{B}\right\rangle$ are $S$-groups by Theorem 7 in [8], and so $A$ and $B$ are SKT modules by Theorem 3.1.

Corollary 3.2 will not only consolidate Wick's study of ch-pure projectives, but it will also be important in our study of these nontrivial mixed $I B$ modules. Often an SKT module can be recognized as an isotype submodule $H$ of a balanced projective group $G$ with a special quotient $G / H$. We will soon see that a $p$-local group $H$ is an SKT module if and only if $H$ appears as an isotype submodule in a balanced projective group $G$ with $G / H$ a coproduct of $c$-valuated countable torsion groups when $G / H$ is endowed with the coset valuation.

THEOREM 3.3. Suppose $\lambda$ is a limit ordinal and $H$ is an isotype $\lambda$-dense submodule of the p-local balanced projective group $G$ which has length $\lambda$. If $\operatorname{cof}(\lambda)=\omega$, then $H$ is balanced projective and is isomorphic to $G$, and if $G / H$ is torsion (regardless of the cofinality of $\lambda$ ), then $H$ is an SKT module.

Proof. When $\operatorname{cof}(\lambda)=\omega$, then any submodule $K$ is $\aleph_{0}$-separable in $G$ if it satisfies $H \subset K \subset G$ since $H$ is $\lambda$-dense. Since $G$ is balanced projective, it has a $K$-nice composition series

$$
0=N_{0} \subset N_{1} \subset \cdots \subset N_{\alpha} \subset \cdots \subset G,
$$

and from this we can form the $\aleph_{0}$-separable composition series

$$
H=N_{0}+H \subset N_{1}+H \subset \cdots \subset N_{\alpha}+H \subset \cdots \subset G
$$

over $H$. It follows that $H$ is balanced projective by Theorem 1.2. Since $H$ and $G$ clearly have the same Ulm-Kaplansky and Warfield $h$-invariants, we also have $H \cong G$. 
Now suppose $\operatorname{cof}(\lambda) \geq \omega$ and $G / H$ is torsion. Since $H$ is $\lambda$-dense in $G, G / H=$ $\bigoplus D_{i}$, where each $D_{i} \cong Z\left(p^{\infty}\right)$ and the direct sum is a valuated coproduct with each nonzero element of $G / H$ under the coset valuation assuming the limit value $\lambda$. For each $i$, select a $\lambda$-elementary $S$-group $K_{i}$ embedded as an isotype $\lambda$-dense subgroup of the totally projective group $G_{i}$ so that $G_{i} / K_{i} \cong D_{i}$ as $c$-valuated groups when $G_{i} / K_{i}$ is endowed with the coset valuation. Now set $K=\bigoplus K_{i}$ and $G^{\prime}=\bigoplus G_{i}$, and observe that $G / H \cong G^{\prime} / K$ as $c$-valuated groups endowed with the coset valuation. By Corollary 2.3, $H$ is isomorphic to a summand of the SKT module $B \oplus K$, where $B$ is balanced projective and $K$ is an $S$-group, and so $H$ is also an SKT module by Corollary 3.2.

THEOREM 3.4. A p-local group $H$ is an SKT module if and only if $H$ appears as an isotype submodule of a p-local balanced projective group so that $G / H$ is a coproduct of countable torsion c-valuated groups when $G / H$ is endowed with the coset valuation.

PROOF. Since the stated condition is clearly necessary (since each $S$-group appears as an isotype subgroup of a totally projective group with cokernel a d.s.c.), we will write $G / H=\bigoplus_{I} C_{i}$, where each $c_{i}$ is a countable torsion $c$-valuated group, and show that $H$ is an SKT module. By Theorem 2.8 in [4], there is an isotype subgroup $K_{i}$ of a totally projective group $G_{i}$ such that $G_{i} / K_{i} \cong C_{i}$ in the category of $c$-valuated groups when $G_{i} / K_{i}$ is endowed with the coset valuation. Moreover, with $G^{\prime}=\bigoplus G_{i}$ and $K=\bigoplus K_{i}$, it is clear that $G / H \cong G^{\prime} / K$ by a map preserving coset valuations. By Corollary 2.3, $H$ is isomorphic so a summand of $K \oplus B$ for some balanced projective group $B$. But $K$ is necessarily an $S$-group since each $K_{i}$ is by Theorem 2.11 in [4], so we conclude that $H$ is an SKT module by Corollary 3.2 .

Theorem 3.4 is not as strong as it would appear since there exists an SKT module $H$ which appears as an isotype submodule of a balanced projective group $G$ such that $G / H$ is torsion-free. Furthermore Hill and Megibben have produced an example (5.3 in [4]) of an $S$-group $H$ which appears as an isotype subgroup of a totally projective $p$-group $G$ such that $G / H$ is a reduced totally projective group of length exceeding $\omega_{1}$. Anomalies such as these are typically constructed when one begins with a $c$-valuated group $A$ having a particular structure and then uses Theorem 1.1 to embed an isotype submodule $H$ in a balanced projective $G$ with $G / H \cong A$ as $c$-valuated groups when $G / H$ is endowed with the coset valuation.

We consider the $p$-local divisible group $Q$ as being generated by elements . ., $x_{-i}$, $x_{-i+1}, \ldots, x_{0}, x_{1}, \ldots, x_{i}, \ldots$ such that $p x_{j}=x_{j-1}$ for all $j \in Z$. Hence if $Q$ is $c$ valuated with the maximum valuation, then every element has value $\infty$. There are two other types of $c$-valuations one can place upon $Q$. The first is where every nonzero element is assigned the limit value $\lambda$, and the second is where there exists some $j$ such that $\left|x_{j}\right|=\lambda+1,\left|x_{i}\right|=\lambda$ for all $i>j$, and $\left|x_{i}\right|=\lambda+k$ for all $i<j$ with $k=j-i+1$. We will call these nonmaximum $c$-valuated $Q$ 's as being of type $\lambda$ and $\lambda+1$, respectively. If $C_{1}$ and $C_{2}$ are two $c$-valuated $Q$ 's of type $\lambda+1$, then clearly $C_{1} \cong C_{2}$ by a map preserving valuations, and so we will speak of this $Q$ as being the unique (up to isomorphism) $c$-valued $Q$ of type $\lambda+1$. This analysis of $c$-valuated $Q$ 's is perfectly analogous to Hill and Megibben's treatment of $c$-valuated $Z\left(p^{\infty}\right)$ 's [4]. 
PROPOSITION 3.5. If $H$ is an isotype submodule of the p-local balanced projective group $G$ such that when endowed with the coset valuation $G / H$ is a c-valuated $Q$ of type $\infty$ or $\lambda+1$, then $H$ is an SKT module.

PROOF. If $G / H$ is the maximum valuation $c$-valuated $Q$, then $H$ is balanced projective since it is a summand of $G$, so suppose $G / H$ is a $c$-valuated $Q$ of type $\lambda+1$. Select a $\lambda$-elementary $S$-group $K$ which is embedded as the torsion part of the $\lambda$-elementary balanced projective group $G^{\prime}$. Now $G^{\prime} / K$ is a $c$-valuated $Q$ of type $\lambda+1$ when endowed with the coset valuation, and so by Corollary 2.3, $H \oplus B \cong K \oplus B$ for some balanced projective group $B$. It follows that $H$ is an SKT module by Corollary 3.2.

Of course, $H$ will be an SKT module if it appears as an isotype submodule of a balanced projective group $G$ so that $G / H$ is a coproduct of $c$-valuated $Q$ 's having type $\lambda+1$ for various limit ordinals $\lambda$. A natural question remains which pertains to the structure of $H$ if $G / H$ is a $c$-valuated $Q$ of type $\lambda$. Is $H$ also an SKT module? The answer to this question is in the negative as our next example shows.

EXAMPLE 3.6. $H$ cannot be an SKT module if it appears as an isotype subgroup of a balanced projective group $G$ so that $G / H$ endowed with the coset valuation is a $c$-valuated $Q$ of type $\lambda$ with $\operatorname{cof}(\lambda)>\omega$.

PROOF. First observe that if there exists one SKT module $H$ such that $G / H$ is $Q$ of type $\lambda$, then any such $H$ will also be an SKT module by Corollary 2.3 and Corollary 3.2. So clearly if such an example exists, where $H$ is not SKT and $G / H$ is a $Q$ of type $\lambda$, then it can come from Theorem 1.1. To prove that this $H$ is not SKT requires a closer analysis of Hill and Megibben's proof of Theorem 1.1. For each nonzero $x \in Q$ we choose $N_{x}=\bigoplus_{\gamma<\lambda} p^{\gamma} M_{\gamma}, G_{x}=\bigoplus_{\gamma<\lambda} M_{\gamma}$, and $\pi_{x}: N_{x} \rightarrow Q$ mapping each $p^{\gamma} M_{\gamma}$ onto the cyclic group $\langle x\rangle$. Here $M_{\gamma}$ is a $\gamma$-elementary balanced projective for $\gamma$ ranging over all limit ordinals less than $\lambda$. Now set $N=\bigoplus_{0 \neq x} N_{x}$ and $G=\bigoplus_{0 \neq x} G_{x}$, and observe that $\pi$ induced by all the $\pi_{x}$ 's can be lifted to an epimorphism $\phi: G \rightarrow Q$ since $G / N$ is totally projective and $N$ is nice in $G$. Finally, $H=\operatorname{ker} \phi$ is isotype in $G$ and $Q \cong G / H$ as $c$-valuated groups when $G / H$ is endowed with the coset valuation. Therefore $H$ is $\lambda$-dense in $G$, but actually more can be said from our construction; namely that $G=H+\left(p^{\alpha} G \cap N\right)$ for each $\alpha<\lambda$.

Now suppose $H=K \oplus S$, where $K$ is balanced projective and $S$ is an $S$-group. Now $K$ is isotype in $G$, and since $K$ is balanced projective, $K$ is complete in its $\lambda$-topology since it is the direct sum of modules having length less than $\lambda$. (Recall that the $\lambda$-topology is formed by taking $p^{\alpha} K$ for each $\alpha<\lambda$ as a neighborhood subbasis about the origin.) Choose any $z \in N$ which is not in $H$, and for each $\alpha<\lambda$ select $h_{\alpha} \in H$ and $g_{\alpha} \in\left(p^{\alpha} G \cap N\right)$ such that $z=h_{\alpha}+g_{\alpha}$. Now each $h_{\alpha} \in H \cap N$, and so each $h_{\alpha} \in K$ since $h_{\alpha}$ has no gaps in its height sequence. Hence $\left\{h_{\alpha}\right\}_{\alpha<\lambda}$ yields a Cauchy net in the $\lambda$-topology on $K$ since

$$
\left|h_{\beta}-h_{\alpha}\right|=\left|\left(z-h_{\beta}\right)-\left(z-h_{\alpha}\right)\right|=\left|g_{\beta}-g_{\alpha}\right| \geq \beta
$$

for every $\beta \leq \alpha$ and since $K$ is isotype in $G$. Since $K$ is complete, the net $\left\{h_{\alpha}\right\}$ converges to $k$ for some $k \in K$, and so $\left|h_{\alpha}-k\right|=\alpha$ for every $\alpha<k$. But then $|z-k|=\left|\left(z-h_{\alpha}\right)-\left(k-h_{\alpha}\right)\right| \geq \alpha$ for every $\alpha<\lambda$, and so $|z-k|=\lambda$. but this is clearly a contradiction since the coset valuation on $G / H$ assumes only the value $\lambda$ for nonzero cosets. We conclude that $H$ is not an SKT module. 
4. Properties of $I B$ modules. In this final section, we will list properties $I B$ modules share that are not necessarily true for arbitrary $p$-local modules. One can think of these properties as being inherited from the balanced projective. For example, $I B$ modules are transitive, fully transitive, and any two $p^{\alpha}$-high submodules of an $I B$ module are equivalent. A $p$-local group $G$ is transitive if for any two elements $x, y \in G$ with $\left|p^{n} x\right|_{G}=\left|p^{n} y\right|_{G}$ for each $n<\omega$, there exists an automorphism of $G$ carrying $x$ onto $y$, and $G$ is fully transitive if for any two elements $x, y \in G$ with $\left|p^{n} x\right|_{G} \leq\left|p^{n} y\right|_{G}$ for each $n<\omega$, there exists an endomorphism of $G$ mapping $x$ onto $y$. The fact that $I B$ modules are transitive follows immediately from Theorem 2.2 and the fact that $I B$ modules are fully transitive follows from Theorem 2.4.

THEOREM 4.1. IB modules are transitive and fully transitive.

The argument used to prove Theorem 4.1 in [4] will carry over routinely to prove the following proposition.

Proposition 4.2. A p-local group $G$ is an $I B$ module if and only if both $p^{\alpha} G$ and $G / p^{\alpha} G$ are $I B$ modules for some ordinal $\alpha$.

Recall that a submodule $H$ of $G$ is $p^{\alpha}$-high in $G$ for some ordinal $\alpha$ if $H$ is maximal with respect to the property that $H \cap p^{\alpha} G=0$. The study of $p^{\alpha}$-high subgroups is abundant in the literature.

THEOREM 4.3. Any two $p^{\alpha}$-high submodules of an IB module are equivalent.

Proof. Suppose $H$ and $K$ are $p^{\alpha}$-high subgroups of the $p$-local group $G$ which appears as an isotype submodule of the balanced projective group $T$. We can restrict ourselves to the case where $\alpha$ is infinite, and so we write $\alpha=\lambda+n$ for some $n<\omega$ and some limit ordinal $\lambda \geq \omega$. It is well known that under the circumstances $H$ is isotype in $G$ (and hence in $T$ ) with $p^{\lambda} H$ bounded. It is also true, however, that $H$ is $\lambda$-dense in $G$ even though elements of infinite order may occur. Indeed, it is clear that $G\left[p^{m}\right] \subseteq H+p^{\beta} G$ for all $\beta<\lambda$ and all $m<\omega$, so suppose $x \in G$ has infinite order. There will be a nonnegative integer $k$ such that $p^{k} x+h \in p^{\alpha} G$ for some $h \in H$, and since $H$ is isotype in $G$, if $\beta$ is a fixed but arbitrary ordinal $<\lambda$, then $p^{k} x+p^{k} \bar{h}=p^{k} g$, where $\bar{h} \in H$ and $g \in p^{\beta} G$. Hence $x+\bar{h}-g \in G\left[p^{k}\right] \subseteq H+p^{\beta} G$, and so $x \in H+p^{\beta} G$. Since $\beta$ was arbitrary $<\lambda$, it follows that $H$ is $\lambda$-dense in $G$. Noting also that $H$ and $K$ will necessarily have the same Ulm-Kaplansky and Warfield $h$-invariants, we give a sketch of the rest of the proof since it can be completed precisely as in Theorem 4.5 in [4].

We can decompose $p^{\lambda} T=p^{\lambda} H \oplus C$ and $p^{\lambda} G=p^{\lambda} H \oplus B$ with $B=G \cap C$, and since $p^{\lambda} H$ and $p^{\lambda} K$ are both maximal $p^{n}$-bounded summands of $p^{\lambda} G$, it follows that $p^{\lambda} T=p^{\lambda} K \oplus C$ and $p^{\lambda} G=p^{\lambda} K \oplus B$. Now there will be an isomorphism $G / H \rightarrow G / K$ extending the correspondence $b+H \rightarrow b+K$ (for $b \in B$ ), and since $p^{\lambda} T+H=H \oplus C$ and $p^{\lambda} T+K=K \oplus C$, there is an isomorphism $\theta:\left(p^{\lambda} T+G\right) / H \rightarrow$ $\left(p^{\lambda} T+G\right) / K$ which extends the isomorphism $G / H \rightarrow G / K$. Finally we extend $\theta$ to a coset valuation preserving isomorphism $\phi: T / H \rightarrow T / K$, and so $H$ and $K$ are equivalent under an automorphism of $T$ (and hence under one of $G$ ) by Theorem 2.1 and the way $\phi$ was chosen. 


\section{REFERENCES}

1. D. Arnold, R. Hunter and F. Richman, Global Azumaya theorems in additive categories, J. Pure Appl. Algebra 16 (1980), 223-242.

2. P. Hill, On the classificition of abelian groups, Photocopied manuscript, 1967.

3. Verlag, Berlin and New York, 1981, pp. 305-321.

4. P. Hill and C. Megibben, On the theory and classification of abelian p-groups, Math. Z. 190 (1985), 17-38.

5. __ Axiom 3 modules, Trans. Amer. Math. Soc. 295 (1986), 715-734.

6. __ Torsion-free groups, Trans. Amer. Math. Soc. 295 (1986), 735-751.

7. R. Hunter, F. Richman and E. Walker, Warfield modules, Lecture Notes in Math., vol. 616, Springer-Verlag, Berlin and New York, 1977, pp. 87-123.

8. R. Hunter and E. Walker, S-groups revisited, Proc. Amer. Math. Soc. 82 (1981), 13-18.

9. M. Lane, A new characterization for $p$-local balanced projective groups, Proc. Amer. Math. Soc. 96 (1986), 379-386.

10. , The balanced-projective dimension of p-local abelian groups, J. Algebra (to appear).

11. M. Lane and C. Megibben, Balanced projectives and axiom 3, J. Algebra (to appear).

12. F. Richman and E. Walker, Valuated groups, J. Algebra 56 (1979), 145-167.

13. R. Stanton, $S$-groups, preprint.

14. E. Walker, Ulm's theorem for totally projective p-groups, Proc. Amer. Math. Soc. 37 (1973), 387-392.

15. R. Warfield, A classification theorem for abelian p-groups, Trans. Amer. Math. Soc. 210 (1975), 149-168.

16. _ Classification theory of abelian groups. I: balanced projectives, Trans. Amer. Math. Soc. 222 (1976), 33-63.

17. B. Wick, A projective characterization for SKT-modules, Proc. Amer. Math. Soc. 80 (1980), 39-43.

Department of Mathematics, Auburn University, Auburn, Alabama 36849

Current address: M.I.T. Lincoln Laboratory, Group 91, Lexington, Massachusetts 02173 